NEURODEGENERATIVE DISEASES

\title{
Laying the groundwork for remyelination
}

Current therapies for multiple sclerosis (MS) are predominantly

Mice injected daily on days 3-14 postinjury had significantly

more remyelination that could, in theory, reverse more immunosuppressive and used to control inflammatory relapses. A recent publication by Keough et al. provides insight into remyelination, a process advanced forms of MS. The authors discovered that the chondroitin sulfate proteoglycans (CSPGs) found in MS plaques inhibited remyelination, and that blocking the production of CSPGs enabled remyelination in focally damaged spinal cords of mice.

Remyelination occurs when oligodendrocyte precursor cells (OPCs), which are found throughout the central nervous system, migrate to regions of damaged myelin and differentiate into oligodendrocytes. Oligodendrocytes then wrap their cell processes into a sheath around the damaged neuron, creating new myelin.

CSPGs are abundant in the neuronal lesions of patients with MS, and these molecules are known to inhibit axon regeneration after traumatic injury. To recapitulate this CSPG-rich microenvironment, the authors precoated plates with CSPGs. These molecules inhibited murine OPC process outgrowth and rendered many previously identified remyelination-promoting compounds ineffective.
They then screened a library of 245 approved and orally bioavailable compounds to try to identify compounds that could promote remyelination in the presence of CSPGs. None of the compounds was effective, so the authors looked for ways to inhibit CSPG production instead. Peracetylated 4-fluoro- $N$ acetylglucosamine (fluorosamine), a derivative of one of the CPSG building blocks, was used to inhibit the production of CSPGs by acting as an inactive substrate to reduce the activity of a key enzyme involved in the CSPG synthesis pathway. Fluorosamine treatment reduced the amount of CSPG secreted into the media by astrocytes. The matrix deposited by fluorosamine-treated astrocytes was permissive for OPC adhesion and process outgrowth, unlike the matrix deposited by untreated astrocytes.

In mice injected with lysolecithin, which produces focal demyelination, daily intraperitoneal injections of fluorosamine from days 3-7 post-injury reduced the amount of CSPGs and increased the number of mature oligodendrocytes in the lesion. Mice injected daily on days 3-14 post-injury had significantly more remyelination than did vehicle-treated mice.

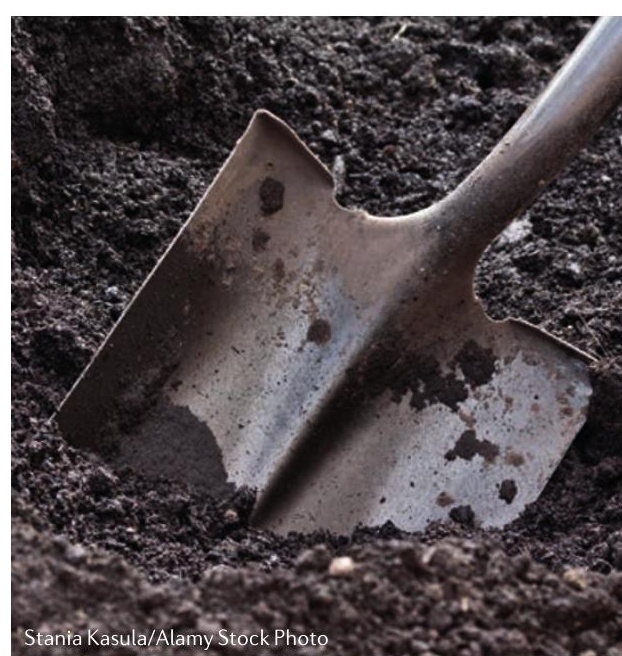

In the experimental autoimmune encephalomyelitis model of inflammatory MS, daily fluorosamine treatment, starting on day 10 (when mice are first symptomatic) or day 15 (when mice have substantial clinical disability) reduced the disease score on days 22-26 relative to vehicletreated mice. Versican, a CSPG, was upregulated in this model, and fluorosamine reduced the versican content in spinal sections.

Combinatorial approaches, in which the production of CSPGs is inhibited while the recruitment and maturation of OPCs is promoted, could have substantial diseasemodifying potential for MS and other demyelinating conditions.

Megan Cully

ORIGINAL ARTICLE Keough, M. B. et al. An inhibitor of chondroitin sulfate proteoglycan synthesis promotes central nervous system remyelination. Nat. Commun. 7, 11312 (2016) 